\title{
ABORDAGEM ODONTOLÓGICA EM PACIENTE PORTADOR DE LEUCEMIA LINFOIDE AGUDA: REVISÃO DE LITERATURA
}

\author{
Pricila Trindade Zocante, Pedro Cardoso Silva, Arlete Gomes Santos Parizi \\ Universidade do Oeste Paulista - UNOESTE, Curso de Odontologia, Presidente Prudente, SP. e-mail: \\ prizocante@hotmail.com
}

\section{RESUMO}

O objetivo deste estudo foi analisar informações encontradas na literatura, que dissertem sobre a necessidade de avaliações e assistências odontológicas em pacientes portadores de leucemias, para que a equipe multiprofissional possa proporcionar ao paciente uma realidade menos agressiva da doença, seja na fase de preparação, tratamento ou manutenção. O presente estudo foi desenvolvido por meio da análise documental da produção bibliográfica obtida nas seguintes bases de dados: Biblioteca Virtual em Saúde (BVS), LILACS, MEDLINE, PubMed e SciELO. A intervenção odontológica reduz a frequência de problemas, minimizando o risco de infecções bucais e complicações associadas. Portanto, a consulta odontológica de um paciente recém-diagnosticado com leucemia deve ser feita o quanto antes, para que haja tempo suficiente para tomar os devidos cuidados antes do início da terapia do câncer. Embora o cirurgião-dentista não seja responsável pelo tratamento do câncer, é importante no tratamento das complicações bucais da doença.

Palavras-chave: leucemia-linfoma linfoblástico de células precursoras, tratamento farmacológico, assistência odontológica, radioterapia, transplante.

\section{DENTAL APPROACH IN PATIENTS WITH LEUKEMIA ACUTE LYMPHOID: LITERATURE REVIEW}

\begin{abstract}
The objective in this study was analyze information found in literature that discusses the need for dental evaluations and assistance in patients with leukemia, so that a multiprofessional team can provide the patient with a less aggressive reality of the disease, treatment or maintenance. The present study was developed through documentary analysis of the bibliographic production obtained in the following databases: Virtual Health Library (VHL), LILACS, MEDLINE, PubMed and SciELO. Dental intervention reduces the frequency of problems, minimizing the risk of oral infections and associated complications. Therefore, the dental appointment of a newly diagnosed patient with leukemia should be done as soon as possible, so that there is sufficient time to take proper care before the start of cancer therapy. Although the dentist not being responsible for treating cancer is important in treating the oral complications of the diseases.
\end{abstract}

Keyword: leukemia-lymphoma precursor cell lymphoblastic, drug therapy, dental care, radiotherapy, transplantation.

\section{INTRODUÇÃO}

A leucemia é uma neoplasia maligna que ocasiona a produção excessiva e constante dos leucócitos no sangue em formato imaturo, sendo esse crescimento desordenado e independente. É um tipo de câncer muito comum em crianças e adolescentes ${ }^{1-4}$.

Geralmente, as leucemias são de origem desconhecida embora alguns estudos apontam que a exposição a grandes doses de radiação ionizante e a produtos químicos como o benzeno, 
infecção por vírus como o Vírus de Epstein-Barr (VEB) e o Vírus linfotrópico humano, bem como condições genéticas como síndrome de Down, neurofibromatose, síndrome de Shwachman e sobrepeso ao nascer podem ser fatores de risco das leucemias ${ }^{3,5,6}$.

As leucemias são classificadas quanto à citomorfologia e o estado de maturação. Quanto à citomorfologia classificam-se em Mieloide quando os mieloblastos levam a supressão medular e em linfoide quando essas células são os linfoblastos. Já de acordo com estágio de maturação classifica-se em aguda ou crônica, ${ }^{5,7}$. Dessa forma, as leucemias podem ser divididas em: leucemia Mieloide crônica (LMC), leucemia Linfoide crônica (LLC), leucemia Mieloide aguda (LMA) e a leucemia Linfoide aguda (LLA) ${ }^{8}$.

A LLA é constituída por células linfoides (glóbulos brancos) imaturas, ocupando um grande espaço na medula óssea onde ocorre sua produção, impedindo assim a produção das plaquetas e glóbulos vermelhos ${ }^{9}$. As leucemias agudas podem ser classificadas por uma variedade de formas, incluindo a morfologia, a citoquímica, os marcadores de superfície, a citogenética e a expressão de oncogenes ${ }^{10}$. É a neoplasia maligna hematológica mais prevalente de infância, ${ }^{3,8,10-13}$, com uma maior incidência entre os dois e cinco anos de idade, sendo $25 \%$ de todos os cânceres nessa faixa etária ${ }^{9,14}$.

O diagnóstico é dado a partir da realização do mielograma, com análise morfológica complementada pelos exames de imunofenotipagem, citogenética e biologia molecular $^{11}$. De modo geral, o tratamento envolve quimioterapia em longo prazo ${ }^{15-17}$, podendo ainda, ter como opções: cirurgias e radioterapia, que podem resultar em reações indesejadas ao paciente, como efeitos adversos e sequelas ${ }^{18}$.

A terapia antineoplásica traz diversas complicações, sendo as lesões bucais uma das principais ${ }^{1,19,20}$. Dados da literatura mostram que cerca de $40 \%$ dos pacientes oncológicos apresentam repercussões bucais agudas, como mucosite, aftas, candidíase, herpes, gengivites, hemorragias gengivais, infecções fúngicas, virais e bacterianas, xerostomia, alterações no paladar, distúrbios na formação dos germes dentários e trismo muscular ${ }^{1,5,19,21}$, além de vômitos, náuseas, diarreia, mucosite gastrointestinal, eritema, erupções, maculo papilar, dermatite, entre outras patologias ${ }^{9}$.
Tais complicações bucais podem comprometer $\mathrm{o}$ protocolo de tratamento quimioterápico tornando, eventualmente, necessária a diminuição da dose administrada, a modificação no protocolo de tratamento ou até mesmo, a interrupção da terapia antineoplásica, afetando diretamente a sobrevida do paciente ${ }^{22}$.

As manifestações bucais podem ser prevenidas, tratadas ou controladas por um cirurgião-dentista especializado na área de estomatologia ${ }^{20}$, possibilitando minimizar essas alterações com cuidados odontológicos prétratamento oncológico, eliminando fatores de risco preexistentes. Idealmente, o cirurgiãodentista deveria estar envolvido desde a época do diagnóstico inicial, preferencialmente como parte da equipe multiprofissional ${ }^{23}$.

Nota-se que a atuação do profissional da Odontologia dentro da equipe multiprofissional que lida com o tratamento antineoplásico é imprescindível, tanto nas fases iniciais de diagnóstico, quanto durante a terapia, realizando avaliações estomatológicas e dando ao paciente condições de ser submetido às modalidades terapêuticas com as melhores possibilidades de cura, prevenindo ou reduzindo os efeitos colaterais $^{5}$.

O objetivo neste estudo foi levantar informações na literatura, que dissertem sobre a necessidade de avaliações e assistências odontológicas em pacientes portadores de leucemia linfoide aguda, para que a equipe multiprofissional possa proporcionar ao paciente uma realidade menos agressiva da doença, seja na fase de preparação, tratamento ou manutenção.

\section{METODOLOGIA}

Este estudo foi desenvolvido por meio da análise documental da produção bibliográfica obtida nas seguintes bases de dados: Biblioteca Virtual em Saúde (BVS) - Bireme, LILACS, MEDLINE, PubMed, SciELO e na base Periódicos Capes. Para as buscas foram utilizados os descritores: "Leukemia-Lymphoma Precursor Cell Lymphoblastic", "Chemotherapy", "Dental Care", "Radiotherapy" e "Transplantation". Adicionalmente, foi realizada busca manual na literatura nacional de periódicos de temas inerentes às leucemias e saúde bucal. Os critérios de seleção dos artigos foram determinados de acordo com o objetivo do presente estudo e considerando artigos disponíveis online, publicados entre 2006 e 2018 na língua portuguesa, inglesa ou 
espanhola. A busca foi realizada no período entre 2015 e 2018 e foram incluídos artigos publicados como: pesquisas originais, relato de casos e artigos de revisão da literatura. Pesquisas que abordaram temas inerentes a Medicina, condição sistêmica, Odontologia e saúde bucal. Após a análise dos estudos, foram selecionadas 34 referências como base para o desenvolvimento da revisão da literatura.

\section{REVISÃO DE LITERATURA}

O cirurgião-dentista destaca-se entre os profissionais envolvidos na equipe multiprofissional responsável pelo tratamento do paciente oncológico, pela sua capacidade no diagnóstico, prevenção, estabilização e tratamento de alterações na cavidade bucal que comprometem a qualidade de vida do paciente antes, durante e depois da terapêutica antineoplásica ${ }^{24}$.

As alterações causadas pelo tratamento oncológico ilustram o fato de que críticas e regulares avaliações da cavidade bucal, atendimentos odontológicos adequados e atenção com a higiene bucal são obrigatórios em todos os pacientes pediátricos que recebem quimioterapia ${ }^{25}$.

Problemas bucais decorrentes da terapia do câncer aumentam o risco de infecção, tempo de internação, custo do tratamento e impacto negativo no curso e prognóstico da doença. Além de visitas periódicas à enfermaria, o odontólogo na equipe tem o foco em ações preventivas e tratamento odontológico antes, durante e após a quimioterapia e/ou radioterapia. O objetivo do seu trabalho é prevenir infecções, manter a higiene bucal e minimizar os efeitos adversos das terapias anticâncer. Além desses objetivos, a unidade atua como centro de referência para o desenvolvimento de pesquisas ${ }^{26}$.

A intervenção odontológica reduz a frequência de problemas, minimizando o risco de infecções bucais e complicações associadas. Portanto, a consulta de um paciente recémdiagnosticado deve ser feita o quanto antes, para que haja tempo suficiente para tomar os devidos cuidados antes do início da terapia do câncer ${ }^{27}$.

Segundo o Instituto Nacional do Câncer (INCA), os problemas bucais já existentes devem ser tratados antes do tratamento oncológico para evitar complicações bucais e evitar dificuldades durante e após o tratamento ${ }^{28}$, tendo em vista que após iniciado o tratamento, esses pacientes passam a ser mais susceptíveis às alterações agudas e crônicas em tecidos moles além de apresentarem um maior risco de osteorradionecrose ${ }^{24}$.

O odontopediatra deve coletar informações sobre doenças adjacentes, tempo de diagnóstico, modalidades de tratamento que o paciente recebeu desde 0 diagnóstico (quimioterapia, radioterapia, cirurgias etc), e complicações, incluindo recaídas, hospitalizações, atendimentos em pronto-socorro, infecções (bucais e sistêmicas), estado hematológico atual, alergias, medicamentos e uma revisão de sistemas (coração, pulmões, rins etc) deve ser solicitado ${ }^{27}$

Faz-se necessária a avaliação por um cirurgião-dentista e a resolução do máximo possível de problemas bucais antes de ser iniciado o tratamento contra o câncer. Nesse contexto, é na ocasião da primeira consulta odontológica que o profissional realiza o exame clínico da cavidade bucal e por meio dele investiga fatores de risco para elaborar um plano de tratamento odontológico capaz de preparar o paciente antes de receber a quimioterapia e a radioterapia ${ }^{24}$.

O ideal seria o emprego de uma abordagem odontológica conservadora, assim como em pacientes hígidos, porém nestes pacientes muitas vezes isso não é possível, pois é necessário realizar a adequação do meio bucal, eliminando não só os focos de infecção ativos como também aqueles que podem se exacerbar com o início da radioterapia ${ }^{29}$.

O paciente deve ter a condição de saúde bucal estabilizada antes do início da terapia antineoplásica, de modo que, ao iniciar o tratamento, o paciente passe a ser monitorado principalmente no que diz respeito às complicações bucais decorrentes da terapia, sejam elas relacionadas à mucosa bucal, à redução salivar ou à combinação dos fatores ${ }^{24}$.

Pacientes submetidos à quimioterapia apresentam-se imunossuprimidos e, portanto, susceptíveis a infecções sistêmicas. Os cuidados odontológicos durante a quimioterapia estão relacionados à manutenção de ótima saúde bucal, tratar quaisquer efeitos colaterais que podem ocorrer devido à terapia antineoplásica e reforçar ao paciente a importância de uma ótima saúde bucal para reduzir problemas/desconfortos durante o tratamento quimioterápico ${ }^{21}$.

No paciente de alto risco (leucemia ativa ou em fase de supressão de medula óssea), a intervenção odontológica é limitada ao cuidado 
de emergência e quando há evidências de infecção bucal, o paciente deve receber antibiótico de largo espectro por via endovenosa. No paciente de risco moderado (fase de manutenção), o tratamento odontológico deve ser evitado no período de 14 dias após o ciclo de quimioterapia por ser o momento de maior mielossupressão. Depois de iniciado o ciclo, o tratamento pode ser realizado antes ou depois de 21 dias da sessão quimioterápica ${ }^{21}$. O oncologista deverá ser consultado antes de qualquer procedimento, incluindo profilaxia ${ }^{30}$.

Durante a radioterapia devem-se tomar medidas como o controle e orientação de higiene bucal e uso de flúor tópico; exodontias são contraindicadas $^{31}$. 0 aconselhamento nutricional é importante para diminuir a perda de peso e desidratação, recomendando o consumo de alimentos líquidos e pastosos em temperatura ambiente, evitando-se dieta cariogênica ou irritante para a mucosa, como temperos, frutas cítricas, refrigerantes e bebidas com alto teor de ácido ${ }^{32}$.

Métodos utilizados para estimular o fluxo salivar podem ser indicados como uso de balas ou chicletes sem açúcar, excetuando aromas irritantes como hortelã e canela, e bochechos com soluções salinas e alcalinas ${ }^{32}$.

Geralmente, os tratamentos para mucosite bucal são apenas paliativos, objetivando diminuir os sintomas e mantendo o controle de quadros infecciosos e/ou hemorrágicos. Dessa forma, os mais comuns são: anestésicos tópicos, drogas anti-inflamatórias, antimicrobianos tópicos e sistêmicos, bochechos com corticoides e colutórios não alcoólicos, crioterapia e fitoterápicos como a camomila ${ }^{33}$. Em nenhuma hipótese devem-se utilizar soluções que contenham álcool ou fenol em sua composição, pois danificam a mucosa e provocam dor ${ }^{30}$ Atualmente a fotobiomodulação, também conhecida como laser de baixa intensidade ou de baixa potência, tem se mostrado eficiente na prevenção e no reparo das lesões, pois proporciona a cicatrização e a diminuição do processo inflamatório, sendo não invasivo e de fácil execução ${ }^{19}$.

A saúde bucal dos pacientes oncopediátricos deve ser monitorada rotineiramente mesmo após a conclusão das terapias antineoplásicas. Nesse sentido, procedimentos mais invasivos devem considerar o contexto da saúde sistêmica do paciente e sempre serem debatidos com os demais integrantes da equipe de assistência à saúde. Assim, aqueles procedimentos que não foram considerados prioridades antes das terapias antineoplásicas, podem ser planejados para serem realizados ${ }^{24}$. É sugerido que seja realizada profilaxia antibiótica durante procedimentos cirúrgicos bucais e maxilofaciais por, pelo menos, seis meses após a conclusão da quimioterapia ${ }^{21}$.

Os objetivos do tratamento odontológico posterior a terapia antineoplásica são o de manter uma excelente saúde bucal; o de reforçar ao paciente e familiares da importância de se manter os cuidados bucais para a vida toda; e o de tratar os efeitos adversos que ocorram a longo prazo na cavidade bucal e no complexo maxilofacial ${ }^{34}$. Os pacientes que foram curados da leucemia são considerados de baixo risco e podem ser atendidos normalmente durante o tratamento odontológico ${ }^{21}$.

\section{DISCUSSÃO}

A literatura mostra a correlação entre os tratamentos oncológicos e as lesões bucais, e a magnitude desses efeitos dependem de uma série de fatores relacionados ao tratamento, ao tumor e ao paciente. O tipo e o grau da malignidade, a dose das drogas utilizadas, a duração da quimioterapia, a idade, diagnóstico e o nível de higiene bucal, antes e durante a terapia, são fatores determinantes para a gravidade das complicações bucais ${ }^{4}$. Portanto, a consulta odontológica em paciente recém diagnosticado, deve ser feita de uma só vez, para que haja tempo suficiente para que os cuidados sejam concluídos antes do início da terapia oncológica ${ }^{29}$.

Vários artigos corroboram no que diz respeito em medidas iniciais no tratamento odontológico em pacientes portadores de leucemia como identificar e estabilizar ou eliminar reais ou potenciais pontos de infecção e áreas de irritação na cavidade bucal, sem atrasar desnecessariamente $\mathrm{o}$ início do tratamento oncológico; entrar em contato com a equipe profissional responsável pelo tratamento antineoplásico no intuito de saber da condição de saúde sistêmica do paciente, bem como do planejamento e tempo de tratamento e; informar o paciente e sua família sobre a importância da saúde bucal para minimizar os desconfortos antes, durante e após o tratamento, assim como as possíveis sequelas imediatas ou tardias da terapia na cavidade bucal e no complexo craniofacial $^{10,31}$. 
Ressaltam também, os devidos cuidados no condicionamento durante $\mathrm{o}$ tratamento oncológico dos pacientes, incluindo controle e orientação de higiene bucal ${ }^{29,32}$, uso de flúor tópico e exodontias que são contraindicadas nesse momento ${ }^{32}$. A intervenção odontológica fica limitada ao cuidado de emergência ${ }^{10,29}$, sob cobertura antibiótica ${ }^{9,29}$ e, o tratamento odontológico deve ser evitado no período de 14 dias após o ciclo de quimioterapia, podendo ser feito antes ou depois de 21 dias $^{10,20,27}$.

Referente à atenção necessária no póstratamento oncológico, ou na fase de manutenção, temos relatadas: visitas periódicas a cada três meses no primeiro ano, e a cada seis meses elas devem ser rigorosamente seguidas, informar aos pais sobre os possíveis efeitos colaterais da quimioterapia nos dentes, mucosa bucal e complexo craniofacial $^{29}$. Ainda, as intervenções odontológicas devem levar em consideração o estado de saúde geral e ser debatida com os outros profissionais que acompanham o caso, e quando for realizar, fazêlas sob cobertura antibiótica por até seis meses após o término da quimioterapia ${ }^{10}$.

De modo geral, quando se estabelece um determinado tratamento, deve ser pensado que impacto o mesmo exercerá em todos os aspectos da vida do paciente. Existe uma relação entre a qualidade de vida e a saúde bucal, dessa forma, devem ser tomadas medidas que visem minimizar os danos causados pelo tratamento estabelecido. Dessa forma, tornam-se necessários os conhecimentos do profissional sobre essa relação entre a qualidade de vida, saúde bucal e o tratamento indicado ao paciente $^{32}$.

Espera-se que, as informações levantadas nesse trabalho somem e enriqueçam o conhecimento dos cirurgiões dentistas sobre a abordagem odontológica em pacientes com LLA, tanto no pré-, trans- e pós-tratamento neoplásico, oferecendo assim, uma realidade menos agressiva da doença e de suas complicações.

\section{CONCLUSÃO}

A partir desta revisão foram levantadas várias informações que discutem e comprovam a importância de um cirurgião dentista dentro da equipe oncológica. Fica claro que, mesmo não sendo o profissional responsável pelo tratamento do câncer, a presença do odontólogo na equipe multiprofissional pode garantir o tratamento das complicações bucais da doença e de seus tratamentos, oferecendo melhores condições e menores riscos ao paciente.

\section{CONFLITO DE INTERESSE}

Os autores declaram não haver qualquer potencial conflito de interesse que possa interferir na imparcialidade deste trabalho científico.

\section{REFERÊNCIAS}

1. Costa SS, Sousa HR, Costa IS. O papel do cirurgião-dentista no diagnóstico precoce da leucemia e sua responsabilidade ética e legal revisão de literatura. Rev Bras Odonto Legal. 2017;4(2):65-77.

DOI:

http://dx.doi.org/10.21117/rbol.v4i2.82

2. Souza A, Favero L. Uso do brinquedo terapêutico no cuidado de enfermagem à criança com leucemia hospitalizada. Cogitare Enferm. 2012;17(4):669-75.

DOI:

http://dx.doi.org/10.5380/ce.v17i4.30364

3. Morais EF, Lira JAS, Macedo RAP, Santos KS, Elias CTV, Arruda-Morais MLS. Oral manifestations resulting from chemotherapy in children with acute lymphoblastic leukemia. Braz J Otorhinolaryngol. 2014;80:78-85. DOI: http://dx.doi.org/10.5935/1808-8694.20140015

4. Juarez-Lopez MLA, Solano-Silva MN, FragosoRios R, Murrieta-Pruneda F. Oral diseases in children with acute lymphoblastic leukemia with chemotherapy treatment. Rev Med Inst Mex Seguro Soc. 2018;56(2):132-5.

5. Gazzinelli LB, Costa BAO, Gonçalves CF, Junior LAVS, Santos PSS. Manejo odontológico em crianças com leucemia aguda sob tratamento antineoplásico. Rev Uningá. 2018;55(1):121-33.

6. Cordero GB, Hernández OG, Sojo CV. Clinical diagnosis of acute lymphoid leukemia-T. Rev Cienc Méd Pinar Rio. 2017;21(4):545-50.

7. Moraes, AB. Importância do diagnóstico acurado em casos de leucemia mieloide: distinção das leucemias e processos reacionais. [Monografia]. Maringá: Centro Universitário de Maringá, 2017.

8. Santos GDM. Estudo da severidade da mucosite oral em crianças com leucemia 
linfoblástica aguda em hospital de referência em Natal-RN. [Monografia]. Natal: Universidade Federal do Rio Grande do Norte; 2016.

9. Cavalcante MS, Rosa ISS, Torres F. Leucemia linfoide aguda e seus principais conceitos. Rev Cientifica FAEMA. 2017;8(2)151-64. DOI: http://dx.doi.org/10.31072/rcf.v8i2.578

10. Hernández C. Leucemia mieloide aguda diagnóstico, estudio y tratamiento. Man Pract Medicas - Hosp Hermanos Ameijeiras, 2006.

11. Nehmy RMQ, Brito AC, Mota JAC, Oliveira $B M$. A perspectiva dos pais sobre a obtenção do diagnóstico de leucemia linfóide aguda em crianças e adolescentes: uma experiência no brasil. Rev Bras Saúde Matern Infant. 2011;11(3):293-99. DOI:

Http://dx.doi.org/10.1590/S1519-

\section{$\underline{38292011000300010}$}

12. Soares AF, Aquino ARL, Carvalho CHP, Nonaka CFW, Almeida D, Pinto LP. Frequency of oral mucositis and microbiological analysis in children with acute lymphoblastic leukemia treated with $0.12 \%$ chlorhexidine gluconate. Braz Dent J. 2011;22(4):312-6.

DOI:

http://dx.doi.org/10.1590/S0103-

64402011000400009 .

13. Tiol-Carrillo A, Enzaldo-de la Cruz P. Prebacute lymphoblastic leukemia: case report and literature review. Rev Odontol Mex. 2017;21(1):54-60.

DOI: https://doi.org/10.1016/j.rodmex.2017.02.012

14. Almeida ALS, Azevedo IC, Carvalho DPRSP, Vitor AF, Santos VEP, Ferreira Junior MA. Clinical and epidemiological aspects of leukemias. Rev Cuba Hematol Inmunol Hemoter. 2017;33(2):114.

15. Brito AC, Capistrano HM, Torres ML, Ramos G, Viana $\mathrm{MB}$, Oliveira $\mathrm{BM}$. Isolated relapse in the oral cavity of a child with t-lineage acute lymphoblastic leukemia. Braz Dent J. 2012; 23(6):711-15.

DOI:

http://dx.doi.org/10.1590/S0103$\underline{64402012000600015}$

16. Lobão DS, Oliveira BM,Massara MLA, Viana $M B$, Nunes L. Condições da cavidade bucal e acompanhamento odontológico de crianças com leucemia linfocítica aguda. Rev Med Minas Gerais. 2008;18(4):25-32.

17. Martin MB, Li CS, Rowland CC, Howard SC, Kaste SC. Correlation of bone age, dental age, and chronological age in survivors of childhood acute lymphoblastic leukaemia. Int J Paediatr Dent. 2008;18(3):217-23.

DOI:

https://doi.org/10.1111/j.1365-

263X.2007.00892.x

18. Galindo JKSN, Arruda MF, Duque TM, Neris CWD. Relação osteorradionecrose e tratamento endodôntico para pacientes oncológicos : Revisão de Literatura. Rev Uninga. 2016;25(1):59-63.

19. Almeida NES, Andrade MC. Estudo das lesões orais em pacientes pediátricos submetidos à quimioterapia. Anais Seminário de Iniciação Científica. 2017;21:2-5.

20. Pels E, Mielnik-Blaszczak M. Oral hygiene in children suffering from acute lymphoblastic leukemia living in rural and urban regions. Ann Agric Environ Med. 2012;19(3):529-33.

21. Zimmermann C. Tratamento odontológico em pacientes com leucemia de acordo com seus índices hematológicos e fase do tratamento antineoplásico - revisão de literatura. [Monografia]. Florianópolis: Universidade Federal de Santa Catarina. 2012.

22. Chauffaille MLLF, Yamamoto M. Classificação das leucemias agudas: citologia, citoquímica, imunofenotipagem. In: Zago MA, Falção RP; Pasquini R. Tratado de Hematologia. São Paulo: Ed. Atheneu, 2013.p.335-42.

23. Santos MG, Silva LCF, Lins CA, Passos DD, Oliveria Neto JN, Santos TS. Fatores de risco em radioterapia de cabeça e pescoço. Rev Gaucha Odontol. 2010;58(2):191-6.

24. Medeiros-Serpa E, Sousa AS. Tratamento odontológico de pacientes pediátricos submetidos à terapia antineoplásica. In: Ribeiro ILA, Valença AMG, Bonan PRF. Odontologia na oncologia pediátrica. 2.ed. João Pessoa: Ed. Ideia; 2018. p. 137-48.

25. Inati A, Akouri G, Abbas HA. A rare aggravation of severe mucositis post chemotherapy in a child with acute lymphoblastic 
leukemia. F1000Research. 2013;2:196. DOI: https://doi.org/10.12688/f1000research.2-196.v1

26. Carrillo C, Vizeu H, Soares-Júnior LA, Fava M, Odone Filho V. Dental approach in the pediatric oncology patient: characteristics of the population treated at the dentistry unit in a pediatric oncology brazilian teaching hospital. Clinics. 2010;65(6):569-73. DOI: http://dx.doi.org/10.1590/S1807$\underline{59322010000600002}$

27. Xavier AM, Hegde AM. Preventive protocols and oral management in childhood leukemia - the pediatric specialist ' $s$ role. Asian pac J cancer prev. 2010;11(1):39-43.

28. Fadel AP. Investigação laboratorial de LLA. AC\&T Científica. 2010;1(2):10.

29. Ghelardi IR, Soares Junior LAV, Santos PSS, Teixeira SS, Matsushita CM, Carrillo CM, et al. A necessidade da avaliação e tratamento odontológico pré-radioterapia. Prat Hosp. 2008;10(58):149-51.

30. Villela MLD, Silva LCP, Santos RM. Protocolo de atendimento odontológico para crianças acometidas por leucemia linfocítica aguda. Arq Bras Odontol. 2014;10(2):28-34.

31. Santos FC. Tratamento odontológico em pacientes com câncer: Revisão sistemática. [Monografia]. Natal: Universidade Federal do Rio Grande do Norte; 2014.

32. Ribeiro Junior $O$, Borba $A M$, Guimarães Junior J. Prevenção e tratamento da mucosite bucal: o papel fundamental do cirurgião-dentista revisão. Rev Clín Pesq Odontol. 2010;6(1):57-62.

33. Maglia DR, Patuzzi E. Incidência de mucosite bucal em paciente submetido ao tratamento da leucemia linfocítica aguda de acordo com a fase de tratamento e a idade. [Monografia]. Porto Alegre: Universidade Federal do Rio Grande do Sul, 2017.

34. Tosin DC. Tratamento odontológico realizado em pacientes com diagnóstico de câncer atendidos no Serviço de Odontologia Oncológica do UNACON do Hospital Geral de Palmas/Tocantins, no período de abril de 2011 a dezembro de 2016. [Dissertação]. São Paulo:
Faculdade de Odontologia da Universidade de São Paulo; 2017. 